\title{
Looking at scenes while searching for numbers: Dividing attention multiplies space
}

\author{
Helene Intraub and Karen K. Daniels \\ University of Delaware, Newark, Delaware \\ AND \\ TODd S. Horowitz AND JEREMY M. WOLFE \\ Brigham and Women's Hospital, Boston, Massachusetts \\ and Harvard Medical School, Boston, Massachusetts
}

\begin{abstract}
Observers tend to remember seeing a greater expanse of a scene than was shown (boundary extension [BE]). Is undivided visual attention necessary for BE? In Experiment 1, 108 observers viewed photographs with superimposed numerals $(2 \mathrm{~s}$ and $5 \mathrm{~s})$. Each appeared for $750 \mathrm{msec}$, followed by a masked interval and a test picture (same, closer up, or wider angled). Test pictures were rated as the same, closer, or wider angled on a 5-point scale. Visual attention was manipulated with a search task: The observers reported the number of 5s (zero, one, or two). The observers performed search only, picture rating only, or both (giving search priority). Search accuracy was unaffected by condition. BE occurred in both conditions but was greater with divided attention. The results were replicated using incidental BE tests (Experiments 2 and 3). We propose that anticipatory representation of layout occurs automatically during scene perception, with focal attention serving to constrain the boundary error.
\end{abstract}

The visual world is continuous, but physiological constraints prevent us from seeing it all at once. Instead, we sample the environment through movements of the head, body, and eyes. How are successive views integrated within a coherent representation of surrounding space? A common error in scene representation, called boundary extension (BE), may provide part of the answer (Intraub \& Richardson, 1989; for a review, see Intraub, 2007). When viewers explicitly attempt to remember the objects, details, and layout of a scene, they tend to remember more than was actually shown. Their mental representations appear to anticipate the continuity of layout inherent in real-world scenes, which may serve to support integration of successive views into a coherent spatial framework (Gottesman \& Intraub, 2002; Intraub, 1997).

BE occurs rapidly and has been observed following a masked interruption to sensory input as brief as $42 \mathrm{msec}$ (an interval commensurate with the duration of a saccade; Dickinson \& Intraub, 2008). Behavioral experiments (e.g., Gottesman \& Intraub, 2002; Intraub, Gottesman, \& Bills, 1998) and neuroimaging research (Park, Intraub, Yi, Widders, \& Chun, 2007) indicate that it is specifically associated with the processing of layout — an aspect of scene structure that continues beyond the edges of a view. BE appears to occur across the life span. Memory tests revealed layout extrapolation in scene memory for individuals ranging in age from 6 to 84 years old (Sea- mon, Schlegel, Hiester, Landau, \& Blumenthal, 2002). Infants as young as 3 months of age exhibited looking preferences, suggesting that they too remember having seen more of a view than was shown (Quinn \& Intraub, 2007). It is tempting to conclude that $\mathrm{BE}$ is a fundamental aspect of scene representation.

However, the extant literature on BE includes only cases in which observers were presented with a single task. In all BE experiments (with the exception of the infant study, of course), observers were explicitly instructed to memorize each scene for a later memory test. However, in the case of our normal day-to-day scene perception, we do not attempt to memorize each scene that comes into view. In fact, the scene through which we navigate is often incidental to the specific task at hand (e.g., we search for street signs and house numbers as we drive through an unfamiliar neighborhood, while houses and terrain roll by). If BE plays a fundamental role in scene perception, it should occur in such situations as well. On the other hand, if it is related solely to the task of memorizing a view, its utility for everyday scene perception would, at best, be limited, and it surely could not play an important role in view integration. The purpose of our research was to determine whether BE would occur when scene memory was neither the sole task nor the primary task of the observer- that is, when a scene is in the background while visual attention is focused on a demanding, unrelated task in the foreground.

H. Intraub, intraub@udel.edu 
Although the effects of dividing visual attention on $\mathrm{BE}$ are not known, there is certainly evidence that dual tasks compromise picture memory. Rowe and Rogers (1975) demonstrated that verbal shadowing of letters while viewers looked at pictures of outline objects caused a decrement in both recall and recognition of the pictures, when compared with a control condition in which viewers simply heard the letters without shadowing. Wolfe, Horowitz, and Michod (2007) have shown that a superimposed visual search task has a greater negative impact on memory for scenes than does an auditory task of equivalent difficulty. Intraub (1984) demonstrated that during rapid serial visual presentation of photographs, allocation of attention to different subsets of pictures within the stream impaired memory for the unattended pictures.

In a somewhat different vein, visual attention has been shown to influence memory for specific objects or picture details under conditions in which viewers certainly remember the overall scene. Striking examples of viewers' inability to detect changes to scenes have been provided using a variety of paradigms (i.e., change blindness; Levin $\&$ Simons, 1997; Simons \& Rensink, 2005). Viewers frequently fail to notice even large changes in a scene unless they happen to focus attention on those areas (Henderson \& Hollingworth, 1999; Hollingworth \& Henderson, 2002). Related research on inattentional blindness (Mack \& Rock, 1998) and inattentional amnesia (Wolfe, 1999) has highlighted the importance of the allocation of visual attention to scenes and other kinds of visual displays. In a particularly striking class of experiments, observers who were required to count the number of passes made by specific actors in a complex ball-passing game failed to notice a task-irrelevant person in a gorilla suit strolling through the scene (Simons \& Chabris, 1999; see also Neisser \& Becklen, 1975).

Thus, redirecting or dividing visual attention has been shown to affect both overall memory and memory for specific details within a given view. We asked whether BE would also be affected when attention was divided. It is possible that without focal attention to a scene, extrapolation beyond the edges does not occur, either because, in the absence of explicit memorization instructions, constructive memory processes are not elicited or because memory for the spatial expanse of the scene will be so poor that when observers err, they simply make random, bidirectional guesses as to boundary location.

Alternatively, it may be the case that BE is the result of an automatic process during scene perception. It is known that layout is rapidly detected (Biederman, 1981; Schyns \& Oliva, 1994); perhaps the continuation of layout is automatically computed as well. Consistent with this idea, foreknowledge of $\mathrm{BE}$ in conjunction with explicit instructions to prevent it has been shown to attenuate but not eliminate the boundary error (Intraub \& Bodamer, 1993). BE has been observed for briefly presented pictures (e.g., $240 \mathrm{msec}$ ) when memory was tested as soon as 42 msec later (Dickinson \& Intraub, 2008; Intraub \& Dickinson, in press; see also Bertamini, Jones, Spooner, \& Hecht, 2005; Intraub, Gottesman, Willey, \& Zuk, 1996). If BE is automatic, it might be unaffected by the demands of a dual task (similar to gist acquisition; see, e.g., Li, VanRullen, Koch, \& Perona, 2002); another possibility is that not only will BE occur, but also, without focal attention to constrain it, the error will be even more expansive when attention is divided.

The goal of the present experiments was to determine whether BE would be affected if the observer was required to engage in an unrelated, demanding search task while viewing photographs of scenes. Many different types of memory tests have been used to assess BE (drawing tasks, scene reconstructions, border adjustment, serial and forced alternative choice; see Chapman, Ropar, Mitchelle, \& Ackroyd, 2005; Daniels \& Intraub, 2006; Intraub, 2002; Intraub, Hoffman, Wetherhold, \& Stoehs, 2006; Mathews \& Mackintosh, 2004; Seamon et al., 2002). Here, we used an explicit boundary rating scale developed by Intraub and Richardson (1989) that has been used in many subsequent experiments (e.g., Bertamini et al., 2005; Gottesman \& Intraub, 2002; Intraub, Bender, \& Mangels, 1992; Intraub et al., 1998; Intraub et al., 1996), because it allows viewers to respond quickly with a rating that characterizes the view as a whole.

Our choice of search task was driven by the need to engage focal attention. This requires a demanding, inefficient visual search task yielding an RT $\times$ set size slope of approximately 25-50 msec/item (Braun \& Sagi, 1990). The standard choice in the literature on dual visual tasks is a spatial configuration search, which requires the observer to discriminate between two stimuli that differ only in the configuration of their common elements, such as Ts and Ls (Braun \& Julesz, 1998; Li et al., 2002). We opted for a search for block $5 \mathrm{~s}$ among block $2 \mathrm{~s}$, which is a particularly demanding spatial configuration task, perhaps because the distractors are mirror images of the targets (Roggeveen, Kingstone, \& Enns, 2004). In addition, this task has already been successfully used in other research focusing on visual attention and picture memory (Wolfe et al., 2007).

In the present series of experiments, on each trial, $2 \mathrm{~s}$ and $5 \mathrm{~s}$ were superimposed on a briefly presented scene, and observers were asked to report on the number of $5 \mathrm{~s}$ present. This number could vary from zero to two, so that observers would continue searching after finding a single target. This was intended to ensure that the search would require the full 750-msec duration of the composite display. We could then compare boundary ratings between conditions in which observers either ignored the search task or focused on the search task. To prevent one set of attention instructions from interfering with the other, independent group designs were used in all the experiments. To verify that the observers were indeed focused on search when it was the primary task, search performance was always compared with that of a baseline search group. The observers in this group received the search instruction and were warned not to let the pictures distract them. This warning (to ignore the pictures) was further supported by the fact that picture memory was never tested. In Experiment 1, viewers had foreknowledge of the boundary rating task and were tested on each trial, whereas in Experiments 2 and 3, the boundary rating task was incidental, and no mention of it was made until after presentation of all the pictures. 


\section{EXPERIMENT 1}

On each trial in Experiment 1, a close-up or wide-angle view of a scene with a superimposed display of red block $2 \mathrm{~s}$ and $5 \mathrm{~s}$ (see Figure 1) was presented for $750 \mathrm{msec}$. For dual-task observers, the primary task was to report the number of $5 \mathrm{~s}$ without allowing the picture to distract them; for memory-only observers, it was to memorize the picture without allowing the numbers to distract them. After a few seconds, the scene reappeared (either the identical view or the alternative view), and boundary memory was tested using the rating procedure developed by Intraub and Richardson (1989). Viewers indicated whether the test view was the same, a little or a lot more close-up, or a little or a lot more wide-angle (5-point scale). We selected the rating measure because it is a simple, rapid response that captures the overall appearance of the view. This type of test would be less likely to put additional strain on memory by giving the viewers the opportunity to make a decision as soon as they viewed the test item.

Boundary ratings also allowed us to test for key patterns of responses that were associated with BE. These involved responses both to test items that were identical to the stimulus and to those that differed by showing either more or less of the scene than did the stimulus (referred to as distractors). On the basis of prior work with views containing centrally located objects on a simple natural background, when $\mathrm{BE}$ occurs, we should observe the following.

1. BE will be greatest in memory for close-up views. The most sensitive test occurs on trials in which a close-up test item is identical to the stimulus view. On average, at test, the identical close-up should be rated as being more close-up than the stimulusindicating that the observer remembered the stimulus as showing more of the scene.

2. In contrast, wide-angle views will be expected to yield less BE than the close-up views or to yield no directional distortion (e.g., Gottesman \& Intraub, 2002; Intraub et al., 1992; Intraub et al., 1998).

3. When pairs of close-up and wide-angle views are used to create distractor conditions to test memory,
BE will produce a response asymmetry (Gottesman \& Intraub, 2002; Intraub et al., 1998; Intraub \& Richardson, 1989). When a close-up view is followed by a wide-angle view at test, the test item will be rated as being more similar to the stimulus than when the order is reversed. This will occur because BE in memory for the close-up stimulus will result in its being remembered as a more wide-angled view, thus causing it to be more similar to the view shown in the wide-angle test picture. When the wide-angle view is presented first, followed by the close-up view, the difference between the members of the pair will be relatively striking.

We expected to obtain all of these results in the memoryonly condition (replicating prior research). The question was whether any or all of these results would occur under conditions in which attention was withdrawn from the pictures.

\section{Method}

\section{Participants}

The observers were 108 students (68 of them female) from the University of Delaware, who were fulfilling an optional research requirement in an introductory psychology course. All signed IRBapproved informed consent forms.

\section{Stimuli}

The stimuli consisted of close-up and wide-angle views of 48 natural scenes (both indoor and outdoor). Twelve red block numbers ( $2 \mathrm{~s}$ and $5 \mathrm{~s}$ ) were randomly arranged on an invisible $6 \times 4$ grid superimposed on each scene. A close-up and wide-angle version of one such scene, along with the superimposed search display, is shown in Figure 1. An additional five pictures were used to provide practice trials prior to each experimental session.
\end{abstract}

\begin{abstract}
Apparatus
Sequences were programmed in MATLAB with the Psychophysics Toolbox (Brainard, 1997; Pelli, 1997) and were presented on a 21 -in. color monitor run by a Dell Dimension DIMXPS (P4/2.8 GHz) computer. Screen resolution was set to $1,024 \times 768$ pixels $\times 32$ bits of color. The observers were seated approximately $45.7 \mathrm{~cm}$ from the monitor. Each picture was presented on a black background, and the dimensions for each picture were $720 \times 480$ pixels, subtending a visual angle of approximately $29^{\circ} \times 20^{\circ}$. Each block number was $24 \times 40$ pixels, with a stroke width of 9 pixels. At the $45.7-\mathrm{cm}$ viewing distance, each letter subtended $1.0^{\circ} \times 1.7^{\circ}$.
\end{abstract}

A

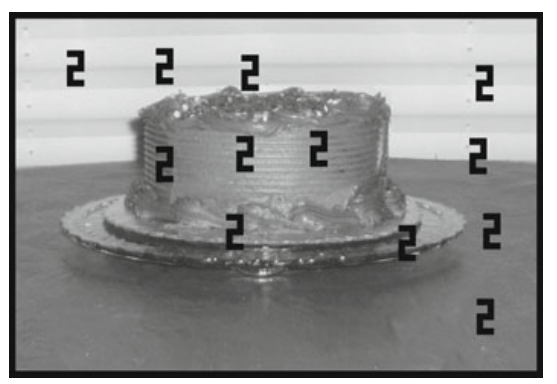

B

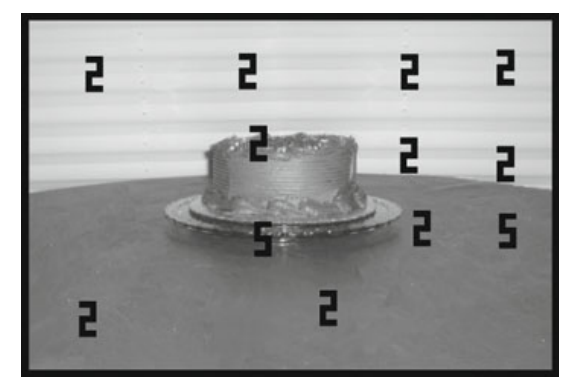

Figure 1. The birthday cake scene is shown in its close-up version with zero target $5 \mathrm{~s}(\mathrm{~A})$ and in its wide-angle version with two target $5 \mathrm{~s}(\mathrm{~B})$. Note that the actual stimuli were presented in color and the search stimuli were in red. 


\section{Design}

The observers were randomly assigned to one of three conditions: memory only, dual task, or baseline search control. There were always 48 trials. Half showed a close-up and half a wide-angle stimulus. The number of target $5 \mathrm{~s}$ was zero, one, or two, equally often. Finally, in the two conditions in which memory was tested, the test view was the same image on half of the trials (wide-wide or closeclose) and the alternate view on the other half of the trials (wideclose or close-wide). Twelve counterbalanced sets of trials were required to ensure that all the scenes were shown equally often in each view with each number of targets and each type of memory test (two stimulus views [close vs. wide] $\times$ three search target quantities [zero, one, or two] $\times$ two test types [same view or different view]). Three observers were tested in each of the 12 counterbalanced sets of trials, so there were 36 observers in each condition and 108 observers in the experiment.

\section{Procedure}

In all the conditions, the stimulus (scene with superimposed numbers) was presented for $750 \mathrm{msec}$, followed by an input task (which timed out after $5 \mathrm{sec}$ ) and a feedback display that remained visible for $2 \mathrm{sec}$. This was done to keep the task situations as similar as possible across conditions. In the memory-only and dual-task conditions, this sequence of events was followed by a boundary rating task (see the Memory Test section below) to assess memory for the picture. In all the conditions, the observers were told the following: "There are always many things in the world that compete for our attention. The purpose of the study is to better understand our ability to pay attention." In the dual-task and baseline search conditions, they were told that their primary task was to count the number of $5 \mathrm{~s}$ in each display without letting the pictures distract them. The memory component of the dual task was described as being of only secondary importance. In the memory-only condition, they were told that their task was to remember the pictures in as much detail as possible without letting the numbers distract them.

Input displays and feedback. In the search conditions (baseline search and dual-task search), the input task involved clicking the number of target $5 \mathrm{~s}$ (zero, one, or two) detected. This was followed by a feedback display that showed "Yes, [\#] targets" following a correct response or "No, not [\#]. There were [\#] targets" following an incorrect response. This display remained visible for $2 \mathrm{sec}$. The input task in the memory-only condition required the observers to click the mouse to report how easy it was to see the picture, on a 5-point scale: very easy (1), fairly easy (2), medium (3), fairly hard (4), or very hard (5). This was followed by a feedback display with the message, "Thank you for your response," which remained visible for $2 \mathrm{sec}$. In all the conditions, if a response was not made within $5 \mathrm{sec}$, the trial timed out, and no response was recorded. In the memory-only and dual-task conditions, the memory test immediately followed. For the baseline search condition only, the feedback display was followed by a $1-\mathrm{sec}$ pause, a ready "beep," and onset of the next trial.

Memory test. Half the time, the test picture that appeared was identical to the stimulus view: a close-up (C) stimulus followed by the same close-up test item (CC condition) or a wide-angle (W) stimulus with the same wide-angle view at test (WW condition). The other half of the time, the test picture was the alternate view: a close-up stimulus followed by wide-angle view at test (CW condition) or a wide-angle stimulus followed by a close-up view at test (WC condition). Following the training procedure in Intraub and Richardson (1989), the impact of moving a camera closer to or farther away from an object in a scene was illustrated to the observers. They were instructed to rate the test items as follows: "Compared to the picture you just saw, this picture is" a lot closer-up $(-2)$, a little closer-up (-1), the same (0), a little farther away (1), or a lot farther away (2). The observers used the mouse to click on their answer; a new screen appeared, and they indicated their confidence by clicking on sure (3), pretty sure (2), or not sure (1). The observers were also given the option of responding did not see picture.

\section{Results and Discussion}

\section{Search Accuracy}

Search accuracy data were arcsine transformed before analysis. We will report the back-transformed mean hit rates. Mean hit rates were above the .33 chance level in both the baseline search condition $[M=.59, S D=.01$; $t(35)=34.75, p<.001]$ and the dual-task condition $[M=.55, S D=.01 ; t(35)=31.17, p<.001]$. Importantly, there was no difference in mean hit rate between the baseline search and the dual-task conditions $[t(70)=$ 1.61, n.s.]. This indicates that the observers in both conditions were equally engaged in the search task.

\section{Memory Task}

Fewer than $1 \%$ of all the responses were lost due to the trial's timing out $(0.4 \%$ in the memory-only condition and $0.5 \%$ in the dual-task condition). The observers rarely reported not remembering a picture at all, but not surprisingly, there were more such responses in the dualtask condition (6.2\%) than in the memory-only condition $(0.4 \%)$.

Table 1 shows the percentage of trials on which the observers reported their confidence as sure (3), pretty sure (2), or not sure (1). Not surprisingly, the observers were more confident in the memory-only condition $(M=$ $2.35, S D=0.32)$ than in the dual-task condition $(M=$ $1.97, S D=0.37)[t(70)=4.61, p<.001]$. Because of this, we analyzed the boundary ratings in two ways: (1) focusing only on high-confidence trials (sure and pretty sure) and (2) analyzing all the trials. Both analyses yielded the same results (with a single exception that will be described later), so we will focus on the high-confidence trials in both conditions.

The critical question in this experiment is whether or not $\mathrm{BE}$ occurred in the dual-task condition. Figure 2A shows that it did. In the CC conditions, in which a close view followed the same close view, the mean boundary ratings were negative, meaning that the observers recalled the first image as having been shown from a wider angle than it actually was. CC boundary ratings were more negative in the dual-task condition than in the memory-only condition

Table 1

Percentages of Sure, Pretty Sure, and Not Sure Confidence Ratings Shown by Condition for Experiments 1-3

\begin{tabular}{lcc}
\hline & \multicolumn{2}{c}{ Condition } \\
\cline { 2 - 3 } Confidence & Memory Only & Dual Task \\
\hline Sure & 42.7 & \\
Pretty sure & 47.1 & 22.1 \\
Not sure & 9.4 & 46.1 \\
& Experiment 2 & 25.1 \\
Sure & 22.9 & \\
Pretty sure & 60.2 & 5.3 \\
Not sure & 15.1 & 35.4 \\
& & 45.6 \\
Sure & 28.7 & 5.1 \\
Pretty sure & 57.6 & 38.2 \\
Not sure & 12.3 & 45.6 \\
\hline
\end{tabular}




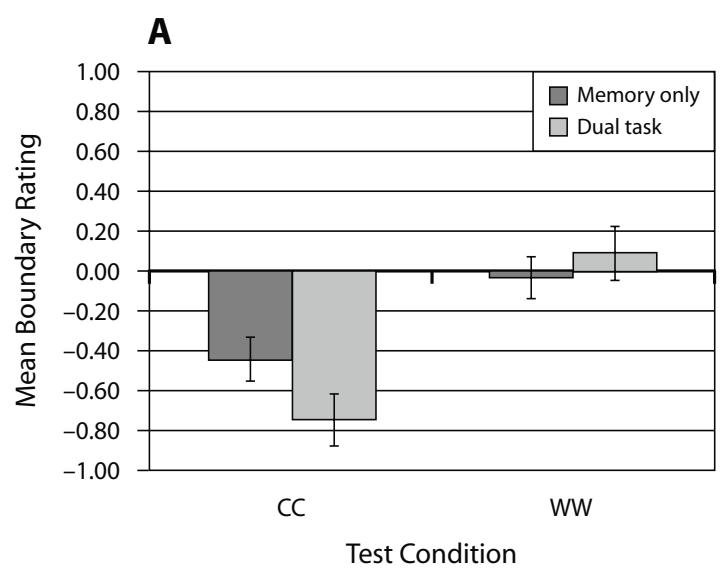

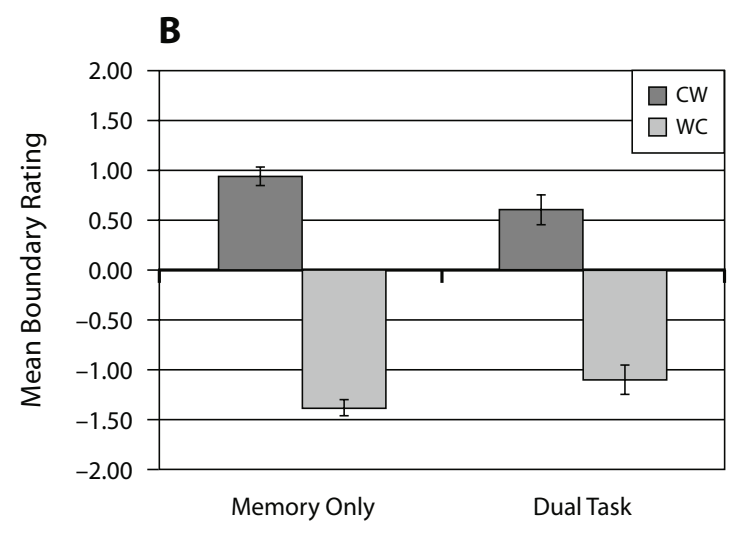

Test Condition

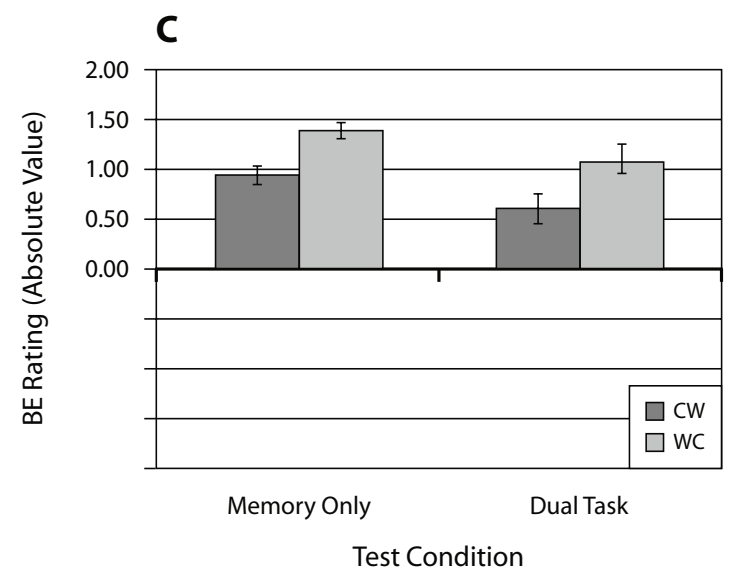

Test Condition

Figure 2. Mean boundary ratings in the memory-only and dual-task conditions in Experiment 1: (A) CC and WW trials, (B) CW and WC trials, and $(C)$ absolute value of the mean ratings on $\mathrm{CW}$ and WC trials (allowing direct comparison of the distractor asymmetry). A rating of 0 indicates that the test item appears identical to the stimulus, negative ratings indicate that it looks more close-up (C) than before (shows less of the scene), and positive ratings indicate that it looks more wide-angle (W) than before (shows more of the scene). Error bars reflect the $\mathbf{. 9 5}$ confidence interval around each mean.

$[t(70)=3.63, p<.001]$. Engagement in the search task had the same effect on BE regardless of the number of targets in the display. For $\mathrm{CC}$ trials in the dual-task condition, mean boundary ratings were $-0.66(S D=0.50)$ in the zero-target condition, $-0.84(S D=0.62)$ in the one-target condition, and $-0.69(S D=0.64)$ in the two-target condition; a one-way ANOVA revealed no significant difference $[F(2,62)=1.01$, n.s.]. As in previous experiments, with single centrally located main objects, no directional error occurred on WW trials in either condition.

It is worth noting that the magnitude of the boundary rating for the $\mathrm{CC}$ trials obtained here is among the highest that have been reported. Across 15 different test conditions in eight publications (using some of the same pictures or pictures very similar to those in the present set),,$^{1}$ the mean rating for CC trials was -0.44 . The highest mean rating in this group $(-0.74)$ was obtained for pictures that were briefly presented (250-msec durations). The briefly presented pictures $(750 \mathrm{msec})$ in the dual-task condition of the present experiment received a similar mean rating.
Figure $2 \mathrm{~B}$ shows the mean boundary ratings for $\mathrm{CW}$ and WC trials. The observers in both conditions were able to correctly recognize that the test picture was a more wide-angle view than was the stimulus on CW trials (mean rating $>0$ ) and a more close-up view than was the stimulus on the $\mathrm{WC}$ trials (mean rating $<0$ ). In addition, these responses show the asymmetry that signifies the presence of BE. To illustrate the response asymmetry, the absolute values of these ratings are presented in Figure $2 \mathrm{C}$. In this way, we can see how much the rating differs from 0 (same) when the same two views are compared but their order of presentation differs. The test item was rated as being more similar to the stimulus view on CW trials than on WC trials. A mixed model 2 (condition: dual task vs. memory only) $\times 2$ (trial type: $\mathrm{CW}$ vs. WC) ANOVA showed that this difference in rating as a function of trial type was significant $[F(1,70)=65.53$, $p<.001]$.

There was also a main effect of condition $[F(1,70)=$ 24.77, $p<.001]$ and no interaction $(F<1)$, indicating 
that responses to distractor views were, overall, less extreme in the dual-task condition. This outcome for $\mathrm{CW}$ trials is consistent with the finding of greater $\mathrm{BE}$ for $\mathrm{CC}$ trials in the dual-task condition, because greater $\mathrm{BE}$ in memory for a close-up stimulus would make it resemble the wide-angle distractor even more (thus, the boundary rating would be expected to be closer to 0 in the dualtask condition). However, the fact that there was also a decrease in WC ratings in the dual-task condition shows that neither type of distractor appeared to be as "different" as it did in the memory-only condition. If this reflects a more conservative use of the scale in the dual-task condition, the observation of greater $\mathrm{BE}$ on $\mathrm{CC}$ trials in the dual-task condition is all the more striking.

We ran a similar experiment in which pictures were counterbalanced across test presentation conditions (CC, $\mathrm{WW}, \mathrm{CW}$, and WC) but the number of targets was randomly assigned to test presentation conditions, rather than being evenly distributed. Given that we found no effect of number of targets on BE in Experiment 1, we will report those results as a replication. In this experiment, $n=24$ in each of the three conditions. As in the previous analysis, we excluded trials rated as not sure in both conditions. Again, the data were normalized using an arcsine transformation of the mean hit rate, and we will report the back-transformed means. Mean hit rates were above chance (.33) in both the baseline search condition $[M=$ $.55, S D=.01 ; t(23)=27.30, p<.001]$ and the dual-task condition $[M=.54, S D=.01 ; t(23)=25.11, p<.001]$. Again, the mean hit rates did not differ $[t(70)=1.61$, n.s. $]$. Figure 3 summarizes the critical results. Panel A shows that, again, $\mathrm{BE}$ for the $\mathrm{CC}$ pictures occurred in both conditions, with significantly more $\mathrm{BE}$ in the dual-task condition $[t(46)=2.46, p<.05]$, and panel B shows that the viewers were able to detect that the test view was different from the stimulus view in the $\mathrm{CW}$ and $\mathrm{WC}$ trials. Panel $\mathrm{C}$ shows the $\mathrm{CW}$ and WC asymmetry characteristic of $\mathrm{BE}$. A mixed model 2 (condition: memory vs. dual task) $\times 2$ (trial type: $\mathrm{CW}$ vs. WC) ANOVA showed a main effect of trial type $[F(1,46)=77.46, p<.001]$, a main effect of

A

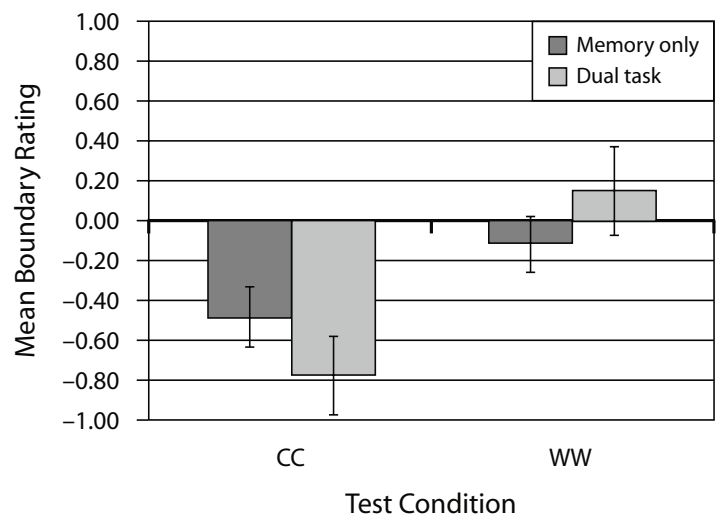

B

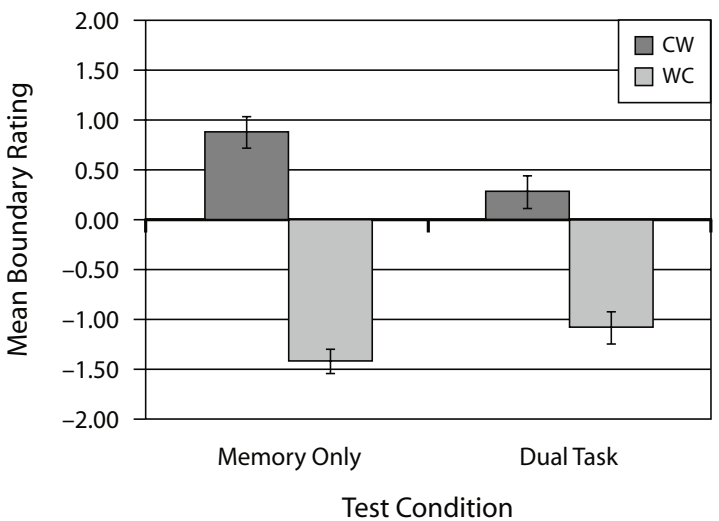

C

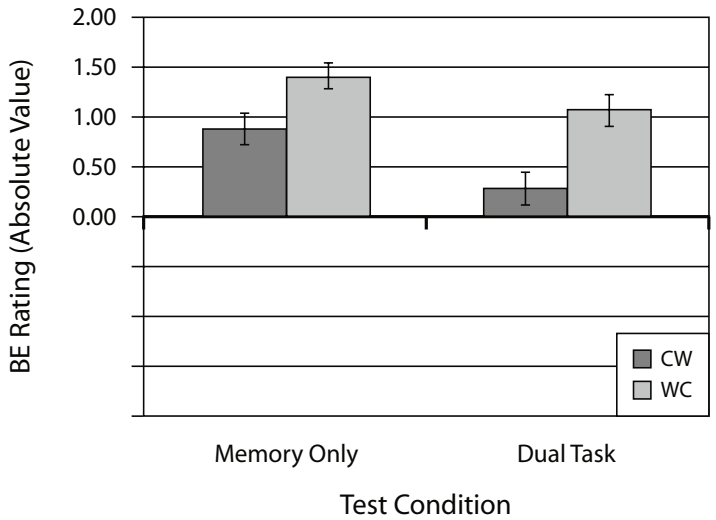

Figure 3. Mean boundary ratings in the memory-only and dual-task conditions in the replication (Experiment 1): (A) CC and WW trials, (B) CW and WC trials, and (C) absolute value of the mean ratings on $\mathrm{CW}$ and WC trials (allowing direct comparison of the distractor asymmetry). A rating of 0 indicates that the test item appears identical to the stimulus, negative ratings indicate that it looks more close-up (C) than before (shows less of the scene), and positive ratings indicate that it looks more wide-angle (W) than before (shows more of the scene). Error bars reflect the .95 confidence interval around each mean. 
condition $[F(1,46)=25.56, p<.001]$, and no significant interaction $[F(1,46)=1.73, p=.19]$.

A data analysis for the primary experiment and for the replication was conducted both including and excluding trials on which the observers expressed low confidence (not sure). When the lowest confidence trials (not sure) were included in the analysis, the only difference we observed was in the case of the WW trials in the dual-task condition of the primary experiment, which yielded significant boundary restriction $[M=.11, S D=.29 ; t(35)=$ $2.22, p<.05]$. A possible explanation is that, in the dualtask condition, low-confidence trials reflected particularly poor memory due to the demands of the search task, causing these responses be more susceptible to proactive interference across trials (in which both close-up and wideangle scenes were presented). As a result, these responses may have been colored by normalization (i.e., regression to the mean across trials, with large objects remembered as being smaller and small objects remembered as being larger; see Intraub et al., 1992).

This pattern did not occur in the replication when not sure trials were added; for WW trials, $M=.04[t(23)=$ 0.51, n.s.]. Finally, although it was not the main purpose of Experiment 2, the possible impact of proactive interference was tested when the number of trials was reduced from 48 to 12 . As will be seen, no boundary restriction occurred for the wide-angle views in the dual-task condition in that experiment.

\section{EXPERIMENT 2}

When the observers were engaged in a demanding primary visual search task, BE clearly occurred for closeups. If anything, the extension was slightly greater than in the absence of a concurrent task. Why might this be? One possibility is that anticipatory representation of layout beyond the edges of a view is the result of a rapid, automatic process that is constrained by focal attention. On the other hand, this might have been due to the implementation of an advantageous strategy in the memory-only condition.

Because they were tested on each trial, the observers in all the conditions were fully aware of the nature of the memory test. The observers in the memory-only condition may have been able to implement strategies that minimized their boundary ratings. For example, although it is highly unlikely, one could verbally quantify the relation of the object to each of the edges of the view; simply characterizing it with respect to a single boundary (e.g., " $0.5 \mathrm{~cm}$ from the right") would serve to minimize the rating. The memory-only group would be more likely than the dualtask group to be able to implement such a strategy. Supporting this possibility is research showing that, at least in the case of a relatively long stimulus duration $(15 \mathrm{sec})$, prior knowledge of the test can attenuate (although not eliminate) BE (Intraub \& Bodamer, 1993).

To tease apart these possibilities, in Experiment 2, we eliminated foreknowledge of the nature of the memory test by deferring the test (and the instructions for the test) until the end of the presentation trials. Because long-term retention of the picture is much more difficult in a dual-task condition, we reduced the number of presentation trials to 12 (from 48). At the end of the 12 trials, boundary memory for the 12 pictures ( 6 close-up and 6 wide-angle) was assessed using the same boundary rating task as that in Experiment 1 . If the difference between the two conditions in Experiment 1 reflected strategic differences in the observers' behavior in the dual-task and memory-only conditions, no difference between groups would be expected in Experiment 2 . However, if the difference between conditions was due to engagement in the demanding search task, we should replicate that difference, using this new procedure.

Finally, because the dual-task observers in Experiment 1 occasionally failed to remember a few pictures, even though memory was tested moments after presentation, given the great memory demands of Experiment 2, we set an a priori criterion: At least three of the six closeups and three of the six wide-angles had to be recognized for a participant's data to be included in the analysis.

\section{Method}

\section{Participants}

The observers were 72 undergraduates (46 of them female) from the University of Delaware, who were fulfilling an optional research requirement in an introductory psychology course and passed our a priori criterion of recognizing pictures on at least 3 of the $\mathrm{CC}$ trials and 3 of the WW trials. Half participated in the memory-only condition, and half in the dual-task condition. A total of 106 students (72 of them female) had participated to accomplish this goal, because 33 observers in the dual-task condition and 1 participant in the memoryonly condition failed to meet these criteria. Data from the first 12 trials of Experiment $1(n=36)$ were used for comparison on the search task. All the observers signed IRB-approved informed consent forms.

\section{Stimuli}

The stimuli included the scenes that were presented in the first 12 trials of Experiment 1 (close-up and wide-angle views) with superimposed block $2 \mathrm{~s}$ and $5 \mathrm{~s}$. The same five practice pictures were used (without the memory test) to familiarize the observers.

\section{Apparatus}

The apparatus was the same as that in Experiment 1, with the following exceptions. Sequences were presented on a 21-in. Nokia color monitor driven by either a Dell Dimension DIMXPS (P4/2.8 GHz) computer or a Dell Optiplex GX270 (P4/2.4 GHz) computer.

\section{Design}

The observers were randomly assigned to either the memory-only condition or the dual-task condition ( $n=36$ each). We compared search accuracy in the dual-task condition with performance on the first 12 trials of the baseline search condition in Experiment $1(n=$ 36), because these trials corresponded to the ones used in this experiment. Scenes were always presented in the same order as that in Experiment 1 (first 12 trials), intermixing close-up and wide-angle views (counterbalanced across observers). Test pictures were always identical to the stimulus picture, so that there were $6 \mathrm{CC}$ trials and $6 \mathrm{WW}$ trials. As before, each combination of picture and presentation test condition (CC and WW) was presented equally often with zero, one, or two target $5 \mathrm{~s}$ in the display. Thus there were six counterbalancing conditions, with 6 observers in each.

\section{Procedure}

The observers experienced 5 practice trials (without the memory test) to familiarize them with their tasks. Attention instructions were identical to those in the corresponding conditions in Experiment 1 , except that the observers were told that a memory test would be administered at the end of all 12 trials and that test in- 
structions would be presented then. We reasoned that the observers would not spontaneously expect a boundary rating test, but to determine whether this was the case, at the end of each session, the observers were asked whether this was the type of memory test that they had anticipated.

\section{Results and Discussion}

\section{Search Accuracy}

As in Experiment 1, hit rates were arcsine transformed, and we will report the back-transformed means. Mean hit rates were above chance $(.33)$ in both the baseline search condition $[M=.59, S D=.03 ; t(35)=19.18, p<.001]$ and the dual-task condition $[M=.62, S D=.05 ; t(35)=$ $16.19, p<.001]$. Again, there was no difference in mean hit rates between the baseline search and the dual-task conditions $[t(70)=-0.70$, n.s. $]$.

\section{Memory Task}

There were very few timeouts $(1.2 \%$ and $1.6 \%$ in the memory-only and dual-task conditions, respectively). The observers who passed the screening criterion failed to recognize a picture on just $0.7 \%$ of the trials in the memory-only condition; this failure rate increased to $12.0 \%$ in the dual-task condition. As before, because boundary scores were not meaningful on these trials, they were excluded from the analysis. The percentage of times the observers in each condition reported being sure (3), pretty sure (2), or not sure (1) is shown in Table 1. Again, the mean confidence rating was higher in the memory-only condition $(M=2.08, S D=0.35)$ than in the dual-task condition $(M=1.53, S D=0.32)[t(70)=$ $7.05, p<.001]$. Given the small number of trials (only 12 , in contrast to the 48 trials in Experiment 1), we did not conduct separate analyses for the high-confidence trials. All the analyses included all three confidence levels.

Figure 4 shows the mean boundary ratings for the $\mathrm{CC}$ and WW trials in the memory-only and dual-task conditions. BE occurred in both conditions on CC trials, and no directional error occurred on WW trials, replicating the typical pattern associated with BE. As in Experiment 1, $\mathrm{BE}$ for $\mathrm{CC}$ trials was reliably greater in the dual-task condition than in the memory-only condition $[t(70)=2.32$, $p<.05]$. Of the 72 observers, only 1 participant in the memory-only condition answered in the affirmative about expecting a $\mathrm{BE}$ rating test; removing this participant did not affect the pattern of results.

Finally, to determine whether our inclusion criterion had inadvertently skewed the data, we analyzed the excluded participants $(n=33)$ separately, with a very loose criterion: at least one recognized picture in the CC condition. In the case of these excluded dual-task observers, even with very thin data, the results were replicated; there was significantly more $\mathrm{BE}(M=-.77, S D=.71)$ in the dual-task condition than in the memory-only condition $(M=-.42, S D=.43)[t(65)=2.50, p<.05]$.

In sum, the results replicated those for the $\mathrm{CC}$ and WW trials in Experiment 1 with an incidental BE test. Although neither group knew the true nature of the memory test, when attention was withdrawn from the

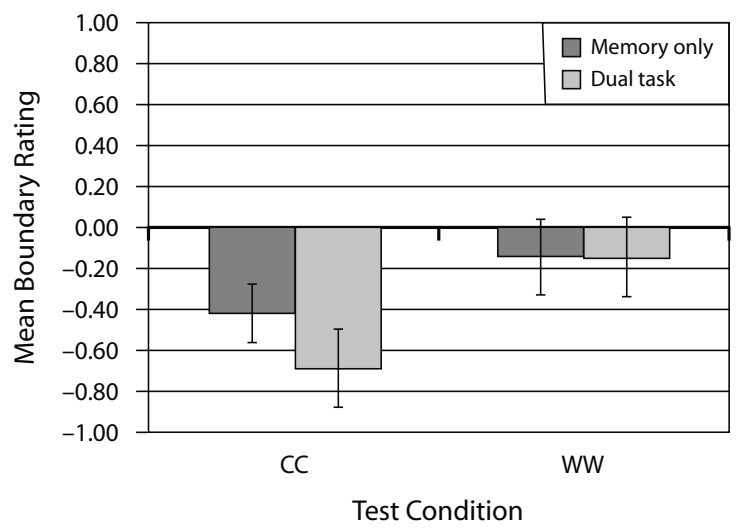

Figure 4. Mean boundary ratings for $\mathrm{CC}$ and $\mathrm{WW}$ trials in the dual-task and memory-only conditions in Experiment 2 (error bars show the .95 confidence interval). C, close-up; W, wide-angle.

pictures, BE was greater in the dual-task condition. This outcome rules out the hypothesis that the difference in BE was due to the use of specific strategies in the memory-only condition in Experiment 1 . The results support the idea that $\mathrm{BE}$ is a rapid, automatic component of scene representation. It does not require focused attention. Indeed, the withdrawal of attention serves only to make the effect larger.

\section{EXPERIMENT 3}

The purpose of Experiment 3 was to replicate the effect of withdrawing attention on BE for close-up views (CC trials) obtained in Experiment 2 while simultaneously testing to determine whether the CW-WC asymmetry, obtained in Experiment 1, would also occur under these conditions. The design was similar to that in Experiment 2, except that the 12 trials always included 6 $\mathrm{CC}$ trials, $3 \mathrm{CW}$ trials, and $3 \mathrm{WC}$ trials. As in Experiment 2 , we set an a priori criterion for recognition. In this case, the viewers needed to recognize at least three pictures in the $\mathrm{CC}$ trials, two pictures in the $\mathrm{CW}$ trials, and two pictures in the $\mathrm{WC}$ trials to be included in the main analysis.

\section{Method}

\section{Participants}

The observers were 72 students ( 39 of them female) from the same population as that in Experiments 1 and 2, who passed our a priori criteria of recognizing at least three $\mathrm{CC}$ pictures, two $\mathrm{CW}$ pictures, and two WC pictures. Half participated in the memoryonly condition, and half in the dual-task condition. A total of 102 students (57 of them female) had participated to accomplish this goal, because 30 observers in the dual-task condition failed to meet this level of performance. As in Experiment 2, data from the first 12 trials of Experiment $1(n=36)$ were used for comparison on the search task.

\section{Stimuli and Apparatus}

Both the stimuli and the apparatus were the same as those in Experiment 2. 
A

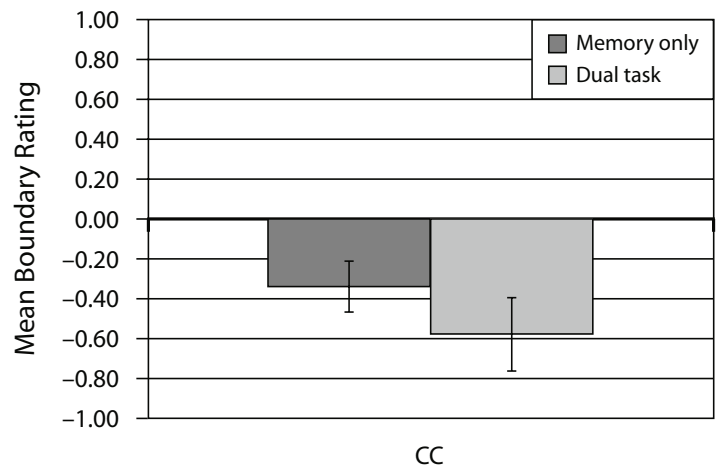

Test Condition

B

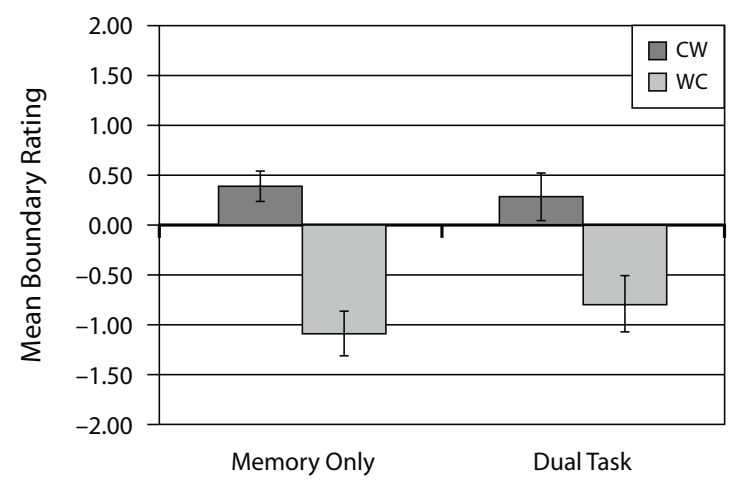

Test Condition

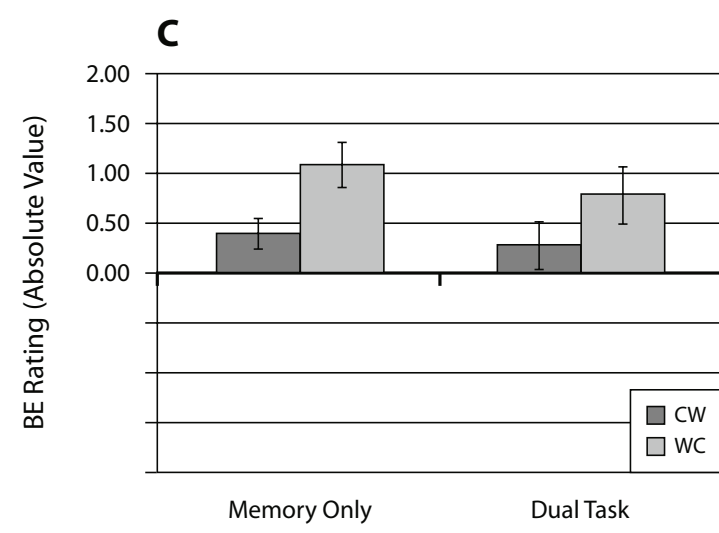

Test Condition

Figure 5. Mean boundary ratings in the memory-only and dual-task conditions in Experiment 3: (A) CC trials, (B) CW and WC trials, and $(\mathrm{C})$ absolute value of the mean ratings on $\mathrm{CW}$ and WC trials (allowing direct comparison of the distractor asymmetry). $\mathrm{A}$ rating of 0 indicates that the test item appears identical to the stimulus, negative ratings indicate that it looks more close-up (C) than before (shows less of the scene), and positive ratings indicate that it looks more wide-angle (W) than before (shows more of the scene). Error bars reflect the .95 confidence interval around each mean.

\section{Design}

The observers were randomly assigned to either the memory-only condition or the dual-task condition $(n=36$ each). As in Experiment 2 , search in the dual-task condition was compared with performance on the first 12 trials in the baseline search condition in Experiment $1(n=$ 36). Scenes were always presented in the same order as in the first 12 trials of Experiment 1, with nine scenes presented in their close-up version and three presented in their wide-angle version. In the memory test, six of the close-ups were tested by repeating the same stimulus (CC trials), three were tested by presenting the more wide-angle version $(\mathrm{CW})$, and the three wide-angle stimuli were tested by presenting a more close-up view (WC). As before, each combination of scene and presentation test condition (CC, $\mathrm{CW}$, and $\mathrm{WC}$ ) was presented equally often with zero, one, or two target $5 \mathrm{~s}$ in the display. Twelve counterbalancing conditions ensured that, across the experiment, each scene was presented half the time in a $\mathrm{CC}$ trial and half the time in a distractor trial (divided evenly between $\mathrm{CW}$ and WC trials). The 12 counterbalancing conditions followed the scheme set out in Experiment 1, except that, instead of having both CC and WW trials, all the trials in which the stimulus and the target were identical were CC trials.

\section{Procedure}

The procedure was the same as that in Experiment 2.

\section{Results}

\section{Search Accuracy}

As in the previous experiments, we arcsine-transformed the search accuracy data and will report the backtransformed means. Mean hit rate for the dual-task condition was above chance $[.33 ; M=.63, S D=.02 ; t(35)=$ $22.78, p<.001]$. This was compared with the same baseline hit rate as that in Experiment 2. Again, the mean hit rate in the dual-task condition did not differ from the baseline rate $[t(70)=-1.15$, n.s. $]$.

\section{Memory Task}

There were very few timeouts $(0.9 \%$ and $1.6 \%$ in the memory-only and dual-task conditions, respectively). For the observers who passed the screening criterion, the failure to recognize a picture was $0.5 \%$ in the memory-only condition and $9.5 \%$ in the dual-task condition. As before, because boundary scores were not meaningful on these trials, they were excluded from the analysis. The percentage of times the observers in each condition reported being 
sure, pretty sure, or not sure is shown in Table 1. Mean confidence was $2.17(S D=0.28)$ and $1.54(S D=0.25)$ in the memory and dual-task conditions, respectively, and differed significantly $[t(70)=10.18, p<.001]$.

Figure 5 shows the critical findings for $\mathrm{BE}$. The mean boundary ratings for the $\mathrm{CC}$ trials in the memory-only and dual-task conditions are shown in panel A. BE occurred in both conditions and was greater in the dual-task condition than in the memory-only condition $[t(70)=2.20, p<.05]$. Panel B shows the mean boundary ratings for the $\mathrm{CW}$ and WC trials. As in Experiment 1, the observers in both conditions were able to correctly recognize that the test picture was a more wide-angle view than was the stimulus on $\mathrm{CW}$ trials (mean rating $>0$ ) and a more close-up view than was the stimulus on the WC trials (mean rating $<0$ ).

To illustrate the distractor asymmetry, panel $\mathrm{C}$ shows the absolute values of the ratings in these trials. Again, the test item was rated as being more similar to the stimulus on $\mathrm{CW}$ trials than on WC trials. A 2 (condition: memory-only vs. dual task) $\times 2$ (trial type: $\mathrm{CW}$ vs. WC) mixed measures ANOVA on the absolute values revealed a significant effect of trial type $[F(1,70)=24.54, p<.001]$ and no interaction $[F(1,70)=1.65$, n.s. $]$. Although the graph suggests that, again, responses to distractors were more muted in the dual-task condition, the main effect of condition in this experiment did not approach significance $(F<1)$.

As in Experiment 2, to determine whether our inclusion criterion had inadvertently skewed the data, we analyzed the excluded observers' boundary scores separately, with the very loose criterion of at least one recognized picture in the $\mathrm{CC}$ condition. We compared their performance with that obtained in the memory-only condition. Again, the excluded observers exhibited significantly more BE $(M=$ $-.60, S D=.69)$ than did those in the memory-only condition $(M=-.34, S D=.36)[t(62)=2.40, p<.05]$.

As in Experiment 2, at the end of the experiment, the observers were asked whether they had expected a memory test like the one administered, and all reported that they had not. These results are contrary to the hypothesis that knowledge of the test led to the development and implementation of a strategy that mitigated BE in the memoryonly condition. As before, when an identical close-up was presented moments later, it did not appear to be the same. Instead, there was a unidirectional error indicating that the observers had remembered the stimulus with extended boundaries. Despite the fact that neither group knew the true nature of the memory test, the size of this effect was greater in the dual-task condition. The distractor asymmetry diagnostic of $\mathrm{BE}$ occurred in both conditions.

\section{GENERAL DISCUSSION}

In three experiments, BE was evident in memory for scenes that had been presented in the background while observers engaged in a demanding, unrelated visual search task presented in the foreground. Dividing visual attention did not eliminate computation of unseen (but highly likely) layout beyond the edges of the view. When visual attention was required for the search task, BE not only oc- curred, but also was more expansive. This shows that BE is not limited to cases in which observers must explicitly memorize the details of a scene and that it is feasible that this aspect of representation may occur during normal perception, when visual attention is often divided.

Whether observers focused on memorizing the briefly presented pictures (ignoring the superimposed numbers) or attempted to count the number of $5 \mathrm{~s}$ among the block $2 \mathrm{~s}$ and $5 \mathrm{~s}$ superimposed on those pictures (a task requiring them to discriminate between two stimuli differing only in the configuration of their common elements), the rating patterns diagnostic of $\mathrm{BE}$ occurred. This occurred whether memory set size was limited to a single picture (Experiment 1) or included 12 pictures (Experiments 2 and 3). In all three experiments, when the stimulus and the test picture were identical close-ups, the test picture was rated as looking too close-up, indicating that the original was remembered as showing a more expansive view, whereas when the stimulus and test pictures were identical wide-angle views, the test picture ratings revealed no directional error. Distractor trials yielded the critical asymmetry showing a unidirectional spatial error. Given the same pair of views, when the close-up was presented first and the more wide-angle view was presented at test, the two were rated as more similar in appearance than when the order was reversed.

In considering our dual-task condition, it is, of course, unwise to assert that a stimulus is processed without visual attention (e.g., Lavie, 1995); however, it is quite clear that our observers had indeed followed instructions and were fully occupied with the demanding visual search task. Accuracy on the search task was well above chance but clearly below ceiling, thus indicating that the observers were engaged in the task but that they found it to be difficult. This level of performance on the primary task (midway between floor and ceiling) is the desired pattern of results and is typical of the results of previous experiments in which what can be processed under conditions of minimal visual attention has been studied (cf. Li et al., 2002). Most important for our purposes, there were no differences between baseline search and search in the dualtask conditions, indicating that dual-task observers gave priority to the search task.

The search task clearly had a negative impact on memory in the dual-task condition. Viewers were more likely to "miss" a picture (i.e., report that they had not seen it before) in the dual-task conditions. This was especially pronounced in Experiments 2 and 3, in which up to 11 trials intervened between presentation and test. These results are consistent with other findings that have indicated that picture memory requires attentional resources (Intraub, 1984; Rowe \& Rogers, 1975; Wolfe et al., 2007). Although fewer pictures were remembered in the dual-task condition, those that were remembered were sufficiently well represented to allow observers to successfully detect distractors and correctly label them as being closer or wider views. This is impressive in that the views were otherwise the same (same object, same background). It is important to note that the ability to identify and classify distractors 
as closer or wider views should not be confused with the critical rating asymmetry for closer-up and wide-angle distractors discussed earlier. The rating patterns observed clearly demonstrated that $\mathrm{BE}$ occurred in the dual-task conditions even though withdrawal of attention had a negative impact on overall memory. In fact, the magnitude of the effect was great even when attention was divided.

In considering the results of Experiment 1, we reasoned that this difference might have been due to the implementation of an advantageous encoding strategy (e.g., noting that "the treetop is $0.5 \mathrm{~cm}$ from the top boundary") that would be much more difficult to exploit in the dual-task condition, because of the demands of the search task. Although it seems unlikely that one could encode such verbal mnemonics for each of the four boundaries, given the brief exposure, correcting even a single boundary in this way would minimize the rating. Thus, knowledge of the test and experience across 48 trials may have resulted in smaller BE ratings in the memory-only condition.

Experiments 2 and 3 tested this possibility by eliminating foreknowledge of the test. Instead of testing after each picture, the observers viewed 12 pictures and then participated in the rating task. The observers were told to try to remember the picture in as much detail as possible and were told that memory would be tested at the end of the presentation series; the nature of the test was not revealed. Most observers tended to expect a recall or recognition test focusing on nameable details about the objects and backgrounds (object identity, colors, type of background, etc.), not a rating scale related to the expanse of the view. (Indeed, out of 144 observers in those experiments, only 1 reported expecting a BE rating test.) In spite of this, the results of Experiments 2 and 3 replicated those of Experiment 1.

One way in which the visual attention manipulation might have had an impact on spatial memory is that the demands of the search task changed the spatial distribution of eye fixations over the image. Although the display duration was brief $(750 \mathrm{msec})$, it is possible that the observers made one or two eye fixations before offset. Because numbers in the search array were never presented at the edges of the picture, the search task may have reduced the number of fixations to the edges of the picture, relative to the center. With fewer fixations on the edge, there may not have been enough detail encoded to support a veridical representation, thus resulting in more $\mathrm{BE}$ in the dual-task condition.

Although eye movements may have had an impact on memory in this way, this account is not as compelling as it might appear at first blush. It has long been known that eye fixations are drawn to high-information areas within a picture, such as objects and object parts (Yarbus, 1967). One can see how, in Experiment 1, with full knowledge of the upcoming boundary test on each trial, the edge of the picture might have become salient enough to draw a fixation; however, the same effect was observed in Experiments 2 and 3 , in which the observers were unaware that a boundary memory test would follow and were instructed to memorize scene details. Given the brief exposure duration of these complex hybrid displays and the expecta- tion of a typical scene memory test, it seems unlikely that fixations would be drawn to the picture's edge, rather than to the main object of the scene. If anything, we would expect fixations away from the edge, since it has been shown that there is a fixation bias toward the center of the screen when observers study scenes (Tatler, 2007).

We favor an alternative explanation that can readily account for the increase in $\mathrm{BE}$ associated with the dualtask condition. We propose that while a scene is being perceived and understood, scene representation includes information derived from sensory activation (sensory), as well as amodal computations beyond the edges of the view (amodal; i.e., amodal perception of surfaces [Kellman, Yin, \& Shipley, 1998; Yin, Kellman, \& Shipley, 2000] or amodal completion of any cropped background objects [Kanizsa, 1979]). These completions help to create a larger spatial framework that captures the sense of place that a view engenders (e.g., we cannot see all four walls in a photograph of a kitchen but do not understand the picture to depict a partial room). While the image is available, the difference between information derived from sensory input and information derived from amodal perception is clearly discernible. However, in memory, the brain does not perfectly tag amodal information as such, so observers are liable to mistakenly remember having seen regions that were not actually present in the image. When the observers must later decide how much of the view they literally saw before, the relative weight of information derived from sensory and amodal information will differ, depending on how much visual attention had been focused on encoding visual details while the image was present.

On this view, deciding how much of a continuous scene one previously saw is analogous to the process of reality monitoring in episodic memory (Johnson \& Raye, 1981). Focused attention to the scene acts to increase the strength of sensory information, causing observers to be less open to accepting increasingly sparse areas of the amodal representation as being information that was actually seen before. Put another way, focused attention raises the threshold for accepting extrapolated layout as having actually been seen. The availability of focused attention reduces $\mathrm{BE}$ by improving memory for what has actually been presented.

Additional support for this interpretation has come from research on the rapidity of BE. As was mentioned earlier, while fixating a picture (sensory input), one can readily report the location of the boundaries. Intraub and Dickinson (in press) demonstrated that a break in sensory input as brief as $42 \mathrm{msec}$ (the duration of a saccade) was sufficient for BE to occur. In one condition, a single scene was presented on each trial. After $250 \mathrm{msec}$ of viewing, the scene was momentarily disrupted by a 42 -msec mask and then remained on-screen. Observers tended to rate the view following the disruption as looking more close-up than the view presented prior to the disruption, indicating that the preinterrupt view was remembered with extended boundaries. Indeed, all the rating patterns diagnostic of BE occurred when $\mathrm{CC}, \mathrm{WW}, \mathrm{CW}$, and $\mathrm{WC}$ conditions were tested in this paradigm. 
Intraub and Dickinson (in press) argued that the most parsimonious explanation for this rapidly occurring error is not to postulate an ad hoc extrapolation process but to consider that, during scene perception, detection of layout automatically evokes a larger spatial framework. This is a graded representation that is most detailed and accurate for foveated information. The representation degrades for more peripheral regions, shading seamlessly into areas of amodal continuation and, ultimately, a more abstract spatial representation. When a reality-monitoring judgment is made at the time of the test, in the absence of sensory input, the observer cannot discriminate between memory for low-acuity sensory input and memory for the amodal continuation of that input. The result is BE.

Other evidence in support of this graded representation view has come from studies in which BE was tested by allowing viewers either to adjust the borders of the view (by moving each one independently with a mouse to reveal more or less of the scene; Intraub et al., 2006) or to adjust the zoom factor of the view, without changing the size of the image (Chapman et al., 2005). In both cases, observers adjusted the test picture to show more than the original stimulus (BE), whether the test picture was identical to the stimulus or showed a greater expanse or a smaller expanse than did the stimulus. However, the amount of extension was greater when the test item was more expansive than when it was less expansive. This may reflect an anchoring issue in making adjustments, but the fact that BE occurred even when the view was identical suggests that it might reflect a graded representation in the following way.

When the test view was identical to the stimulus, viewers "corrected" it to show more (reflecting the influence of the amodal component). When the test view showed a large swath of the "sparse area," viewers tended to accept at least some of it as having been seen before (i.e., they reduced the view, but not enough). When the test view showed less than the stimulus, the "sparse area" and even some of the area derived from sensory information was not present in the test, and without it, the sparse area was not strong enough to be recalled by the viewer. On the basis of recall, the viewer then increased the expanse beyond the edges of the view, but to a lesser degree. The viewers' adjustments in all cases yielded BE, but it was greater when the test view revealed more background than had the stimulus.

Whether or not this particular account of how attention might affect BE is correct, it is clearly the case that engagement in a demanding visual search task does not eliminate BE. If anything, diverting attention increases $\mathrm{BE}$. The computations of layout continuation during scene perception that are ultimately responsible for $\mathrm{BE}$ at the time of the test therefore do not rely on the same limitedcapacity resources as those used in visual search. More likely, BE is produced by parallel processes operating automatically during scene perception.

Although $\mathrm{BE}$ is an error with respect to the stimulus, it is a good prediction of surrounding space that actually does exist beyond the boundaries of the photograph. The tendency for observers to remember seeing beyond the edges of a view captures the continuity of real-world lay- out and may serve to facilitate integration of a given view within a larger spatial framework. The present series of experiments shows that $\mathrm{BE}$ is, at the very least, available to aid integration of successive views even under the rigors of day-to-day perception, wherein viewers do not explicitly attempt to memorize layout and detail but, instead, attend to a task at hand while a scene unfolds around them. If anything, the anticipatory representation of surrounding layout is greater when attention is divided.

\section{AUTHOR NOTE}

This research was supported by NIH Grant R01 MH56020 to J.M.W. We gratefully acknowledge the contribution of research assistants Stephanie Raible, Laura Evans, Carly Nigro, Kari Schweifel, Mattie Wilson, Dan Bensonoff, and David Drowos. Correspondence should be addressed to H. Intraub, Department of Psychology, University of Delaware, Newark, DE 19716 (e-mail: intraub@udel.edu).

Note-Accepted by the editorial board of Editor-Elect Jeremy M. Wolfe.

\section{REFERENCES}

Bertamini, M., Jones, L. A., Spooner, A., \& Hecht, H. (2005). Boundary extension: The role of magnification, object size, context, and binocular information. Journal of Experimental Psychology: Human Perception \& Performance, 31, 1288-1307.

Biederman, I. (1981). On the semantics of a glance at a scene. In M. Kubovy \& J. R. Pomerantz (Eds.), Perceptual organization (pp. 213-263). Hillsdale, NJ: Erlbaum.

Brainard, D. H. (1997). The Psychophysics Toolbox. Spatial Vision, 10, 433-436.

Braun, J., \& Julesz, B. (1998). Withdrawing attention at little or no cost: Detection and discrimination tasks. Perception \& Psychophysics, 60, 1-23.

BraUn, J., \& SAGI, D. (1990). Vision outside the focus of attention. Perception \& Psychophysics, 48, 45-58.

Chapman, P., Ropar, D., Mitchelle, P., \& Ackroyd, K. (2005). Understanding boundary extension: Normalization and extension errors in picture memory among adults and boys with and without Asperger's syndrome. Visual Cognition, 12, 1265-1290.

Daniels, K., \& InTRaub, I. (2006). The shape of a view: Are rectilinear views necessary to elicit boundary extension? Visual Cognition, 14, 129-149.

Dickinson, C. A., \& InTRaub, H. (2008). Transsaccadic representation of layout: What is the time course of boundary extension? Journal of Experimental Psychology: Human Perception \& Performance, 34, 543-555.

Gottesman, C. V., \& Intraub, H. (2002). Surface construal and the mental representation of scenes. Journal of Experimental Psychology: Human Perception \& Performance, 28, 589-599.

Henderson, J. M., \& Hollingworth, A. (1999). The role of fixation position in detecting scene changes across saccades. Psychological Science, 10, 438-443.

Hollingworth, A., \& Henderson, J. M. (2002). Accurate visual memory for previously attended objects in natural scenes. Journal of Experimental Psychology: Human Perception \& Performance, 28, 113-136.

Intraub, H. (1984). Conceptual masking: The effects of subsequent visual events on memory for pictures. Journal of Experimental Psychology: Learning, Memory, \& Cognition, 10, 115-125.

Intraub, H. (1997). The representation of visual scenes. Trends in Cognitive Sciences, 1, 217-221.

InTRAUB, H. (2002). Anticipatory spatial representation of natural scenes: Momentum without movement? Visual Cognition, 9, 93-119.

InTRAUB, H. (2007). Scene perception: The world through a window. In M. A. Peterson, B. Gillam, \& H. A. Sedgwick (Eds.), In the mind's eye: Julian Hochberg on the perception of pictures, films, and the world (pp. 454-466). New York: Oxford University Press.

Intraub, H., Bender, R. S., \& MANGels, J. A. (1992). Looking at pic- 
tures but remembering scenes. Journal of Experimental Psychology: Learning, Memory, \& Cognition, 18, 180-191.

InTRAUB, H., \& Berkowits, D. (1996). Beyond the edges of a picture. American Journal of Psychology, 109, 581-598.

Intraub, H., \& Bodamer, J. L. (1993). Boundary extensions: Fundamental aspect of pictorial representation or encoding artifact? Journal of Experimental Psychology: Learning, Memory, \& Cognition, 19, 1387-1397.

Intraub, H., \& Dickinson, C. A. (in press). False memory 1/20th of a second later: What the early onset of boundary extension reveals about perception. Psychological Science.

Intraub, H., Gottesman, C. V., \& Bills, A. J. (1998). Effects of perceiving and imaging scenes on memory for pictures. Journal of Experimental Psychology: Learning, Memory, \& Cognition, 24, 186-201.

Intraub, H., Gottesman, C. V., Willey, E. V., \& ZuK, I. J. (1996) Boundary extension for briefly glimpsed pictures: Do common perceptual processes result in unexpected memory distortions? Journal of Memory \& Language, 35, 118-134.

Intraub, H., Hoffman, J. E., Wetherhold, C. J., \& Stoehs, S.-A. (2006). More than meets the eye: The effect of planned fixations on scene representation. Perception \& Psychophysics, 68, 759-769.

InTRAUB, H., \& RichaRdSON, M. (1989). Wide-angle memories of closeup scenes. Journal of Experimental Psychology: Learning, Memory, \& Cognition, 15, 179-187.

Johnson, M. K., \& RaYe, C. L. (1981). Reality monitoring. Psychological Review, 88, 67-85.

Kanizsa, G. (1979). Organization in vision. New York: Praeger.

Kellman, P. J., Yin, C., \& Shipley, T. F. (1998). A common mechanism for illusory and occluded object completion. Journal of Experimental Psychology: Human Perception \& Performance, 24, 859-869.

LAVIE, N. (1995). Perceptual load as a necessary condition for selective attention. Journal of Experimental Psychology: Human Perception \& Performance, 21, 451-468.

Levin, D. T., \& Simons, D. J. (1997). Failure to detect changes to attended objects in motion pictures. Psychonomic Bulletin \& Review, 4, 501-506.

Li, F. F., VanRullen, R., Koch, C., \& Perona, P. (2002). Rapid natural scene categorization in the near absence of attention. Proceedings of the National Academy of Sciences, 99, 8378-8383.

MACK, A., \& Rock, I. (1998). Inattentional blindness. Cambridge, MA: MIT Press.

Mathews, A., \& Mackintosh, B. (2004). Take a closer look: Emotion modifies the boundary extension effect. Emotion, 4, 36-45.

Neisser, U., \& Becklen, R. (1975). Selective looking: Attending to visually specific events. Cognitive Psychology, 7, 480-494.

Park, S., Intraub, H., Yi, D.-J., Widders, D., \& Chun, M. M. (2007). Beyond the edges of a view: Boundary extension in human sceneselective visual cortex. Neuron, 54, 335-342.

Pelli, D. G. (1997). The VideoToolbox software for visual psycho- physics: Transforming numbers into movies. Spatial Vision, 10, 437-442.

Quinn, P. C., \& InTraub, H. (2007). Perceiving "outside the box" occurs early in development: Evidence for boundary extension in 3- to 7-month-old infants. Child Development, 78, 324-334.

Roggeveen, A. B., Kingstone, A., \& Enns, J. T. (2004). Influence of interitem symmetry in visual search. Spatial Vision, 17, 443-464.

Rowe, E. J., \& Rogers, T. B. (1975). Effects of concurrent auditory shadowing on free recall and recognition of pictures and words. Journal of Experimental Psychology: Human Learning \& Memory, 1, 415-422.

Schyns, P. G., \& Oliva, A. (1994). From blobs to boundary edges: Evidence for time- and spatial-scale-dependent scene recognition. Psychological Science, 5, 195-200.

Seamon, J. G., Schlegel, S. E., Hiester, P. M., Landau, S. M., \& Blumenthal, B. F. (2002). Misremembering pictured objects: People of all ages demonstrate the boundary extension illusion. American Journal of Psychology, 115, 151-167.

Simons, D. J., \& Chabris, C. F. (1999). Gorillas in our midst: Sustained inattentional blindness for dynamic events. Perception, 28, 1059-1074.

Simons, D. J., \& Rensink, R. A. (2005). Change blindness: Past, present, and future. Trends in Cognitive Sciences, 9, 16-20.

TATLER, B. W. (2007). The central fixation bias in scene viewing: Selecting an optimal viewing position independently of motor biases and image feature distributions. Journal of Vision, 7, 1-17.

WoLfe, J. M. (1999). Inattentional amnesia. In V. Coltheart (Ed.), Fleeting memories: Cognition of brief visual stimuli (pp. 71-94). Cambridge, MA: MIT Press.

Wolfe, J. M., Horowitz, T. S., \& Michod, K. O. (2007). Is visual attention required for robust picture memory? Vision Research, 47, 955-964.

Yarbus, A. C. (1967). Eye movements and vision (Basil Haigh, Trans.). New York: Plenum.

Yin, C., Kellman, P. J., \& Shipley, T. F. (2000). Surface integration influences depth discrimination. Vision Research, 40, 1969-1978.

\section{NOTE}

1. All of the conditions selected made use of the same or similar types of close-up photographs but varied in terms of stimulus duration and the number of stimuli prior to test. The mean is offered simply to provide a point of comparison. Conditions were drawn from Daniels and Intraub (2006), Gottesman and Intraub (2002), Intraub et al. (1992), Intraub and Berkowits (1996), Intraub and Bodamer (1993), Intraub et al. (1998), Intraub et al. (1996), and Intraub and Richardson (1989).

(Manuscript received August 15, 2006; revision accepted for publication April 2, 2008.) 